\title{
Editorial
}

\section{„Virtuelle Realität“ in der publizierten Wissenschaft?}

Für die Wissenschaft ist die Suche nach der Wahrheit oberstes Ziel. Von den Naturwissenschaften nehmen wir an, daß sie besonders exakt sind und die Realität unseres Lebens widerspiegeln. Publizierte Wissenschaft hat demnach Gesetzescharakter. Sie scheint auf unumstößliche Erkenntnisse hinzuweisen, die auf der Suche nach mehr Wissen, nach tieferer Wahrheit und Realität gewonnen wurden. Sie scheint ...?

In den letzten Jahren werden zunehmend Zweifel an dieser Aussage geäußert. Die Editorials zahlreicher großer Zeitschriften beschäftigen sich mit der Qualitätssicherung bei Publikationen (DMW, Rothmund, 1992), mit der Wissenschaftlichkeit medizinischer Publikationen im Brennspiegel (Versicherungsmedizin: Mahr, 1993) und im anglo-amerikanischen Raum sogar mit betrügerischen und überflüssigen Publikationen (JBJS: Bulstrode und Fulford, 1995), mit dem Fehlverhalten in der Forschung (British Medical Journal: Smith, 1996) und der Vorgangsweise bei Betrug (Lancet, 1996). Das Vertrauen in die Wissenschaft ist also offenbar angeschlagen und es ist nicht verwunderlich, daß sich auch die öffentlichen Medien mit den Zweifeln der naturwissenschaftlichen Erkenntnissen beschäftigen.

Anfänglich entstand der Eindruck, daß ein derartiges Fehlverhalten für das nordamerikanische Wissenschaftssystem typisch sei. John Maddox, namhafter Herausgeber der Fachzeitschrift „Nature“ antwortete in einem Interview des Magazins der Frankfurter Allgemeinen Zeitung 1995 auf die Frage: Warum betrügen Wissenschaftler gern?: „In den letzten Zeit - vor allem in den USA und dort in der biomedizinischen Forschung - ist die Versuchung zu betrügerischem Verhalten immer größer geworden. Dies liegt an der besonderen Aufmerksamkeit, die Publikationen als Kriterium für eine Beförderung und die Vergabe von Forschungsgeldern gezollt wird". Anläßlich einer Tagung von Herausgebern naturwissenschaftlicher und medizinischer Fachzeitschriften an der VanderbiltUniversität in Nashville wurde 1992 darauf hingewiesen, daß die Medizin neben der Biologie in dieser Hinsicht besonders anfällig sei. Die Medizin sei eine "großzügige“ Wissenschaft. Die Ergebnisse ließen sich häufig schwer nachprüfen. Biologie und Medizin würden sich daher deutlich von dem Wahrheitsgehalt der physikalischen und chemischen Forschung abheben.

$\mathrm{Da}$ dem nicht so ist, wurde nicht erst durch die Publikationen über die angebliche Kernfusion im Reagenzglas in renommierten Zeitschriften widerlegt. Es wurde aber auch widerlegt, daß diese Probleme ausschließlich auf den nordamerikanischen Markt beschränkt sind. „Ist die Wissenschaft in der Medizin in Gefahr?" lautet der Titel einer Monographie (Schattauer-Verlag, 1993), die zu diesen Problemen auch im deutschen Sprachraum Stellung

Z. Orthop. 134 (1996) 481-482

(C) 1996 F. Enke Verlag Stuttgar nimmt. ,Schummelforscher - Lug und Trug mit Doktorhut -“, so heißt es im Magazin Fokus 1995, in dem auf Fälschungen in der chemischen Grundlagenforschung in der $B R D$ verwiesen wird.

Betroffen macht, daß dieses Problem offenbar seit längerem besteht und nun mitten unter uns ist, auch in der orthopädischen Forschungslandschaft. Die wissenschaftlichen Zeitschriften müssen sich daher mit diesem Problem auseinandersetzen, so auch die Herausgeber der Zeitschrift für Orthopädie.

Wie ist es möglich, daß der Wahrheitsgehalt, die Realität von wissenschaftlichen Publikationen angezweifelt werden kann? - Die fast unübersehbare Zahl von Veröffentlichungen, der Leistungsdruck bei limitierten finanziellen Resourcen für die wissenschaftliche Forschung, aber auch der Leistungsdruck auf dem akademischen Stellenmarkt sind wohl die Hauptursachen. Die Kurzlebigkeit wissenschaftlicher Erkenntnisse macht die Autoren für Datenflälschungen anfälliger. Die moderne Technologie ermöglicht bisher Unbekanntes: Textbausteine können am Computer beliebig zusammengesetzt werden. Abbildungen können modifiziert und komponiert werden. Das Gefällige findet Glauben, das Kontroverse wird eher bezweifelt. Durch die Brille gesehen wird aus der Realität eine virtuelle Realität.

Kann die Wissenschaft, können die wissenschaftlichen $\mathrm{Pu}$ blikationsorgane diese Entwicklungen aufhalten oder müssen wir mit solchen Verfehlungen leben? - Zeitschriften, die wie die Zeitschrift für Orthopädie nach dem Peerreview-Prozeß beurteilen, sollen sicherstellen, daß nur Arbeiten mit ausreichend gesicherten Erkenntnissen publiziert werden. Allerdings werden $85 \%$ der von den besten Journalen abgelehnten Arbeiten letztlich doch trotz aller Mängel veröffentlicht. In diesen Fällen sind die wissenschaftlichen Gesellschaften aufgerufen, sich mit derartigen Problemen zu beschäftigen und Verstöße zu ahnden. In den USA wurden die wissenschaftlichen Gesellschaften zu spät auf die Problematik aufmerksam. Demgemäß hat sich das Gesundheitsministerium der Sache angenommen und Definitionen vorgelegt (s. Kasten). Die Kommission betont, daß der Wahrheitsgehalt der Forschung vor allem dadurch gefördert wird, daß eindeutige Standards für das Verhalten in der Wissenschaft, für die stetige Überprüfung dieser Standards durch Ausbildung und wissenschaftliche Entwicklung vorgegeben werden. Die Kommission kam auch zu dem Schluß, daß die Verantwortlichkeit für die Erhaltung der wissenschaftlichen Integrität und die Aufspürung wissenschaftlichen Fehlverhaltens primär bei den Wissenschaftlern selbst, den wissenschaftlich tätigen Institutionen und wissenschaftlichen Gesellschaften liegen müsse (Smith, 1996). Auch die Wissenschaftler in Deutschland, die orthopädischen Fachgesellschaften und die Zeitschrift für Orthopädie müssen sich mit dem Fehlverhalten in der Forschung auseinandersetzen. Eine Besinnung auf ethische Prinzipien ist in der vermeintlich großzügigen medizinischen Wissenschaft überfällig. Andernfalls könnte es zu einem Vertrauensverlust in der Forschung kommen, der das Ansehen in der Gesellschaft erheblich beeinträchtigt. Ohne Vertrauen kann aber die Wissenschaft, auch die orthopädische, nicht bestehen. 


\section{Fehlverhalten in der Forschung}

Fehlverhalten in der Forschung liegt dann vor, wenn jemand das geistige Eigentum oder Beiträge andere Versionen widerrechtlich als sein Eigentum ausgibt, wenn der Fortschritt der Forschung absichtlich behindert wird, wenn wissenschaftliche Aufzeichnungen verfälscht werden oder wenn die Rechtschaffenheit der wissenschaftlichen Praxis aufs Spiel gesetzt wird. Ein solches Verhalten ist unethisch und für Planung, Durchführung, Berichterstattung, Begutachtung von Forschungsprojekten und Forschungsberichten anderer unzulässig.

Beispiele für wissenschaftliches Fehlverhalten ohne Anspruch auf Vollständigkeit:

\section{Widerrechtliche Aneignung}

Ein Forscher oder Gutachter darf nicht absichtlich oder fahrlässig

a) ein Plagiat begehen: das bedeutet Formulierungen oder Ideen anderer als die seinen auszugeben, ohne entsprechenden Hinweis auf die Quelle.

b) die ihm vertraulich anläßlich der Begutachtung von Anträgen oder Manuskripten zugekommenen Informationen weiter verwenden.

\section{Beeinträchtigung}

Ein Forscher oder Gutachter darf sich das forschungsbezogene Eigentum eines anderen nicht absichtlich bzw. ohne Genehmigung aneignen, beschlagnahmen oder materiell beschädigen. Dies gilt ohne Einschränkung auch für Appataruren, Reagenzien, biologische Materialien, schriftliche Aufzeichnungen, Daten, Hardware, Software und alle im Zuge der Forschung verwendeten oder erzeugten anderen Substanzen oder Geräte.

\section{Ungenaue oder falsche Darstellung}

Ein Forscher oder Gutachter darf nicht in betrügerischer Absicht oder fahrlässig

a) etwas beträchtlich Falsches behaupten oder präsentieren oder

b) eine Tatsache verschweigen, so daß die gemachten Angaben als Ganzes beträchtlich falsch sind.

\section{Literatur}

Bulstrode, Ch., Ph. Fulford: Fraudulent and redundant publication. JBJS 77B (1995) 845-846

Dealing with deception - editorial - The Lancet 347 (1996) 843

Flöhl, R.: Falsche Forschungsergebnisse - schlimmer als gefälschte Frankfurter Allgemeine Zeitung, 1. April 1992

Lehrl, S., E. Gräßel: Wissenschaftliche Autorenschaft: Normen zur Erhöhung der Fairne B. Deutsches Ärzteblatt 90 (1993) 1121-1123

Lug und Trug mit Doktorhut. Fokus 8 (1995)

Maddox, J.: Warum betrügen Wissenschaftler. Interview im Magazin der Frankfurter Allgemeinen Zeitung 1995
Mahr, H.: Wissenschaftlichkeit medizinischer Publikationen im Brennspiegel. Versicherungsmedizin 45 (1993) 173-175

Rothmund, M.: Qualitätssicherung bei Publikationen. Dtsch. Med. Wschr. 117 (1992) 1854-1858

Smith, R.: Time to face up to research misconduct. BMJ 312 (1996) 789-790

F. U. Niethard, Aachen R. Kotz, Wien 